\title{
Consensus of multiple national guidelines: agreed strategies for initial stone management during COVID-19
}

\author{
B. Heijkoop ${ }^{1}$ (I) $\cdot$ E. Galiabovitch ${ }^{1} \cdot$ N. York ${ }^{2} \cdot$ D. Webb ${ }^{1}$
}

Received: 5 July 2020 / Accepted: 7 October 2020 / Published online: 23 November 2020

(c) Springer-Verlag GmbH Germany, part of Springer Nature 2020

\begin{abstract}
Purpose To review the existing available information regarding urolithiasis management and the impact of COVID-19 on this, and propose recommendations for management of emergency urolithiasis presentations in the COVID-19 era.

Methods Review of published guidelines produced by Urological Governing Bodies, followed by the literature review regarding urolithiasis management during the COVID-19 pandemic.

Results Consistent recommendations across guidelines and literature were that urolithiasis with concurrent sepsis or renal failure remains a urological emergency warranting urgent intervention within the pandemic environment. Ureteric stenting and percutaneous nephrostomy are considered equivalent for decompression in this setting, with both ideally to be performed under local anaesthesia where possible to spare ventilators and reduce aerosol-generating procedures. Greater utilization of medical expulsive therapy and dissolution chemolysis may occur during the pandemic, and longer indwelling stent times may be accepted while definite stone clearance is deferred.

Conclusions Urolithiasis will continue to be a source of emergency presentations requiring urgent intervention during the COVID-19 pandemic. However, it is possible to limit these interventions to decompression of the collecting system in the setting of concurrent obstruction or infection, performed under local anaesthesia to limit use of resources and minimise aerosol-generating procedures, with deferral of definitive management.
\end{abstract}

Keywords COVID-19 · Coronavirus · Pandemic · Urolithiasis · Urology

\section{Introduction}

The COVID-19 global pandemic is potentially the most significant challenge faced by healthcare services in the lifetime of today's clinicians, and impacts all specialties. Outside of the direct threat to patients and staff from infection with the virus itself, ramifications of bed and equipment shortages (such as Intensive Care Unit (ICU), ventilator and Personal Protective Equipment (PPE) availability) and delays in diagnosis and management of non-COVID conditions will add

Electronic supplementary material The online version of this article (https://doi.org/10.1007/s00345-020-03491-7) contains supplementary material, which is available to authorized users.

B. Heijkoop

bridgetcheijkoop@gmail.com

1 Austin Health, The University of Adelaide, Heidelberg, VIC, Australia

2 Auckland Regional Urology Service, Auckland, New Zealand additional complexity to management of the situation, and it is likely that alterations to the best practice recommendations will need to be accepted in the short term.

Urolithiasis, and in particular renal colic, is a common emergency urological presentation. Timely management is required to control pain and prevent renal failure secondary to obstruction, and is critical where concurrent urosepsis is present. It is unlikely that the burden of this work will reduce over the duration of the COVID-19 pandemic. Consequently, Urologists must have a plan of action on how best to manage these patients within the limitations of the pandemic environment. While there are multiple established efficacious treatments for urolithiasis, frequently these require anaesthetic, operative and or intensive care support in the perioperative period as well as an inpatient hospital stay. Availability of all these resources may be limited or even unavailable in the pandemic setting. Therefore, decision making regarding most appropriate treatment must consider not only individual patient presentation but also judicious use of these resources. 
Our objective was to review the existing available information regarding urolithiasis management and the impact of COVID-19 on this, and propose recommendations for the initial management of emergency urolithiasis presentations in the COVID-19 era.

\section{Materials and methods}

We first accessed the webpages of major urological governing bodies and clinical guideline institutions including the American Urological Association (AUA), British Association of Urological Surgeon (BAUS), Canadian Urological Association (CUA), European Urological Association (EAU), Urological Society of Australia and New Zealand (USANZ), Societe Internationale d'Urologie (SIU) and National Institute for Health and Care Excellence (NICE), reviewing these for guidelines or position statements directly relevant to Urolithiasis or management of urolithiasis during COVID-19.

We then conducted a non-systematic literature review searching PubMed database with the search terms Urology AND covid-19.

Having summarised the existing information gathered from these sources, we discuss basic principles of working within a global pandemic proposing interim recommendations for emergency management of urolithiasis during the pandemic.

\section{Results: existing information}

\section{Guidelines and position statements: stone management}

Guidelines for urolithiasis management were available from the American Urological Association (AUA), British Association of Urological Surgeons (BAUS), Canadian Urological Association (CUA), European association of Urology (EAU) and NICE [1-6]. Their recommendations are summarised in the table (Table 1). There was concordance between guidelines on fundamental concepts including that concurrent obstructing stone and UTI/urosepsis constitutes a urological emergency and should be managed by urgent decompression with either ureteric stenting or nephrostomy (which were considered of equivalent efficacy), that noncontrast CT is the standard for diagnostic stone imaging, and that NSAIDs should be the first-line analgesic choice for patients with stones. Recommendations regarding the role of conservative management, medical expulsive therapy and stenting were more varied. Notably we were not able to locate published guidelines directly produced by SIU or USANZ.

\section{Guidelines and position statements: COVID-19}

Advice from urological governing bodies regarding urological practice during the COVID-19 pandemic was variable in both quantity and form. The most formal written recommendations from a urological body were produced by the EAU in the document 'EAU Guidelines office rapid reaction group: An organisation-wide collaborative effort to adapt the EAU guidelines recommendations to the COVID-19 era' [7]. In addition, the EAU also provided a link to a collation of all currently published or accepted articles in its partner journal 'European Oncology'. USANZ also published of a total of four sets of specific guidelines [8-11].

Similar to the EAU, CUA published their own written COVID-19 recommendations, as well as providing links to relevant articles published in the Canadian Urological Association Journal (CUAJ) [12]. In addition, a 'Urology Support Webseries' was publicly available from the 'CUA COVID-19 Educational Resources' page of the CUA website, with the recording 'An update from the Canadian Endourology Group (CEG) during the COVID-19 crisis' specifically discussing approaches to stone patients [13, 14]. These recommendations are summarised by Table 2 .

In contrast to the other organisations, the SIU website included only a general statement on COVID-19 without specific guidelines available, and information on the BAUS site was available only to members, requiring a login for access. Finally, the AUA site referenced the recommendations made by other health and government bodies and provided links to relevant articles published in The Journal of Urology, but did not appear to have separately produced any AUA specific guidelines regarding Urological practice during the COVID-19 era in addition to these.

\section{Current literature}

Non-systematic literature review was conducted with most recent review of results on 6/6/2020. Search of PubMed using the terms Urology AND COVID-19 (all fields) returned 243 results. 141 of these were relevant to Urological practice during COVID-19 with 16 reporting findings or making recommendations specific to urolithiasis management [7, 15-29]. Recommendations and findings relevant to urolithiasis in the included articles are summarised in the table (Table 3). With the exception of a single systematic review, included papers were of moderate to low level of evidence including observational studies (2), narrative reviews or publication of expert opinion/recommendations (12) and a case report (1). The included systematic review concerned general urologic manifestations 


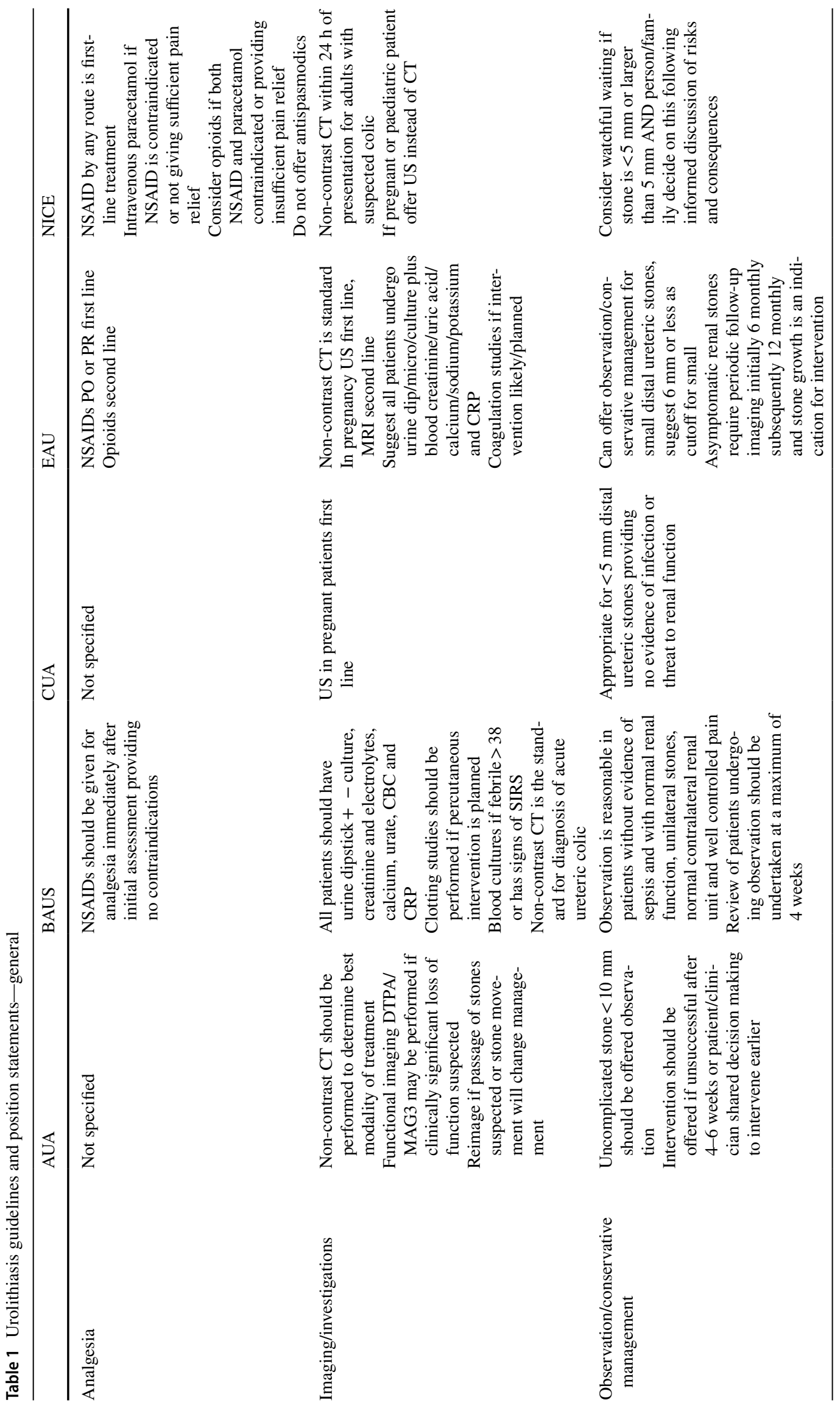




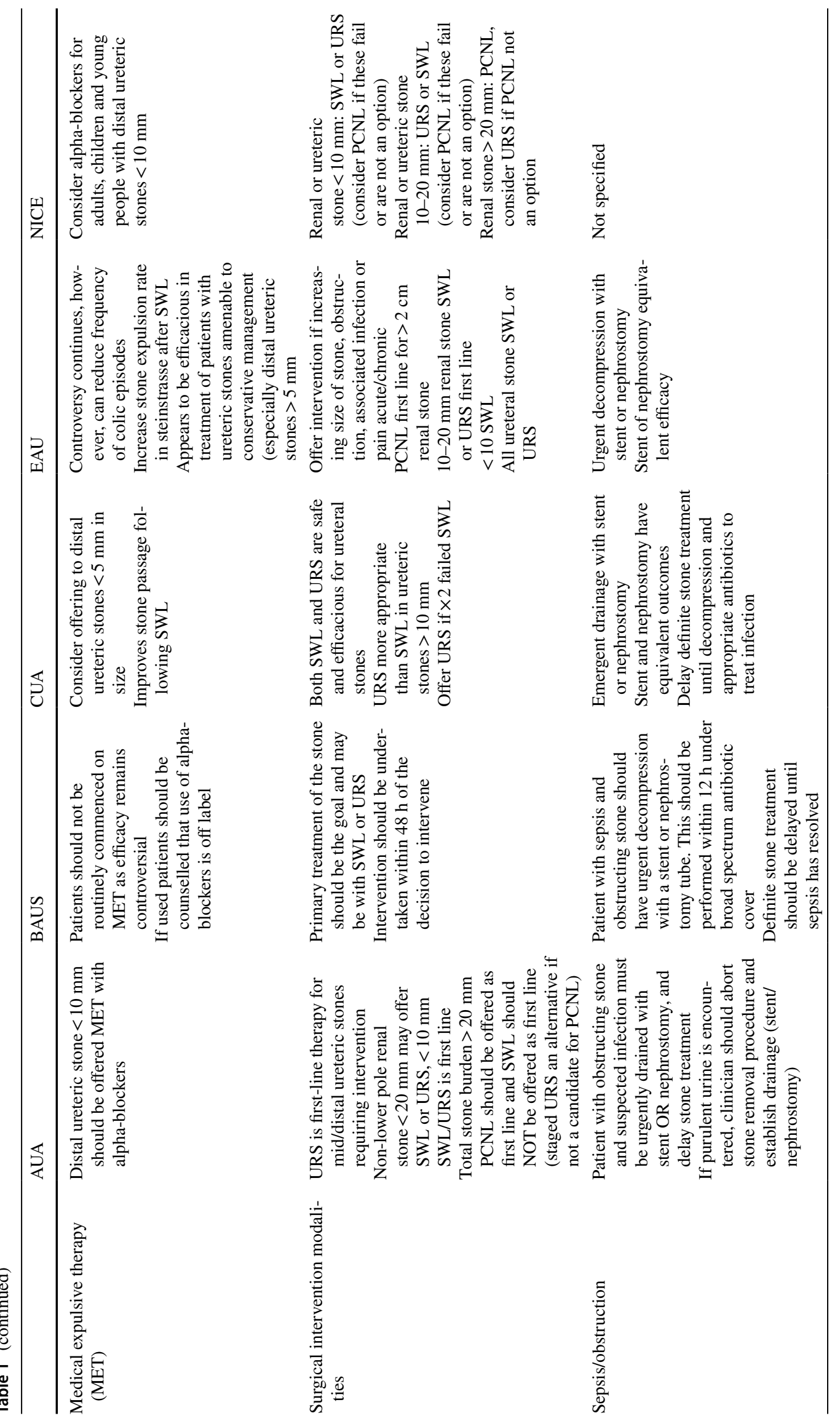




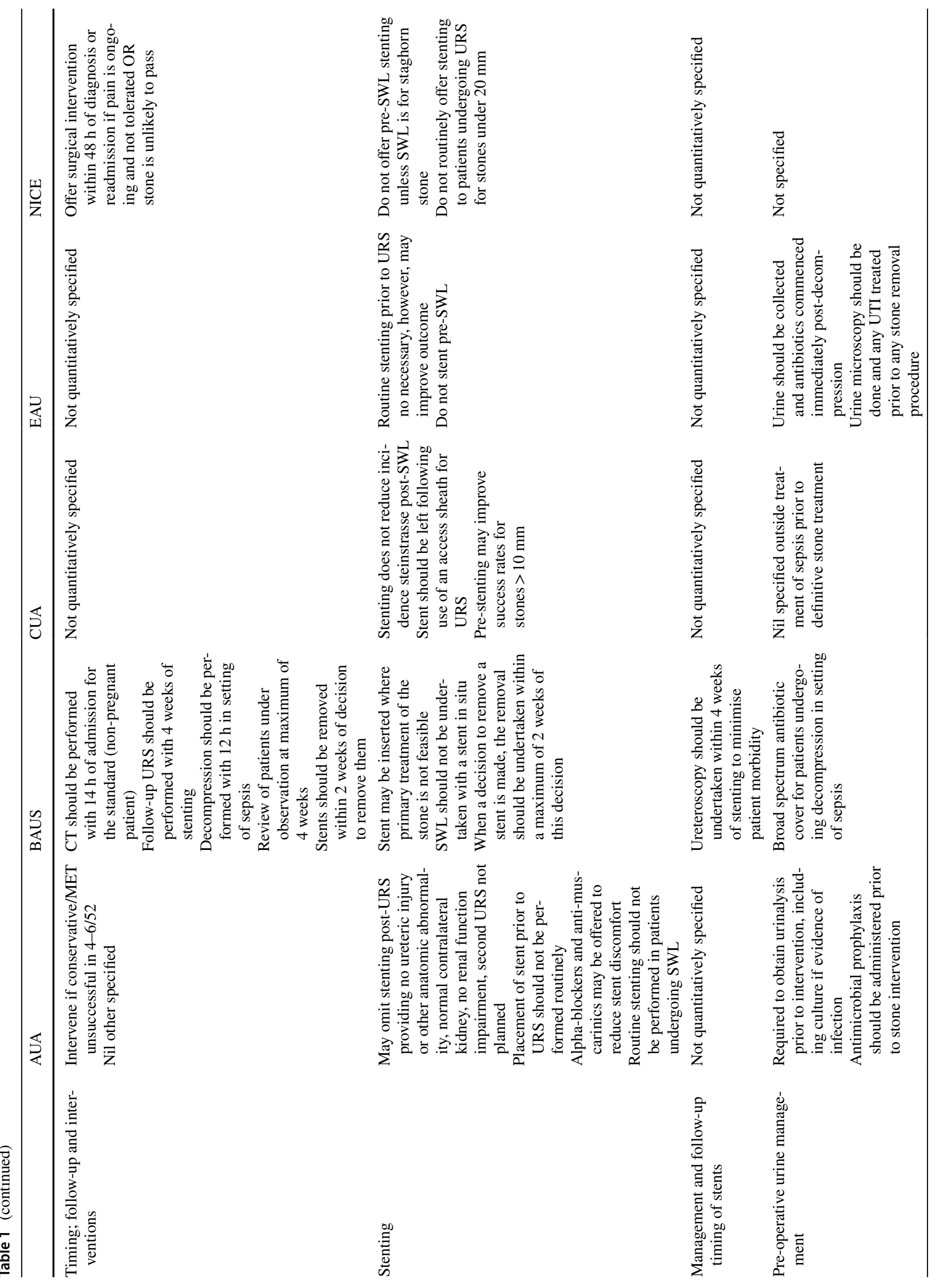




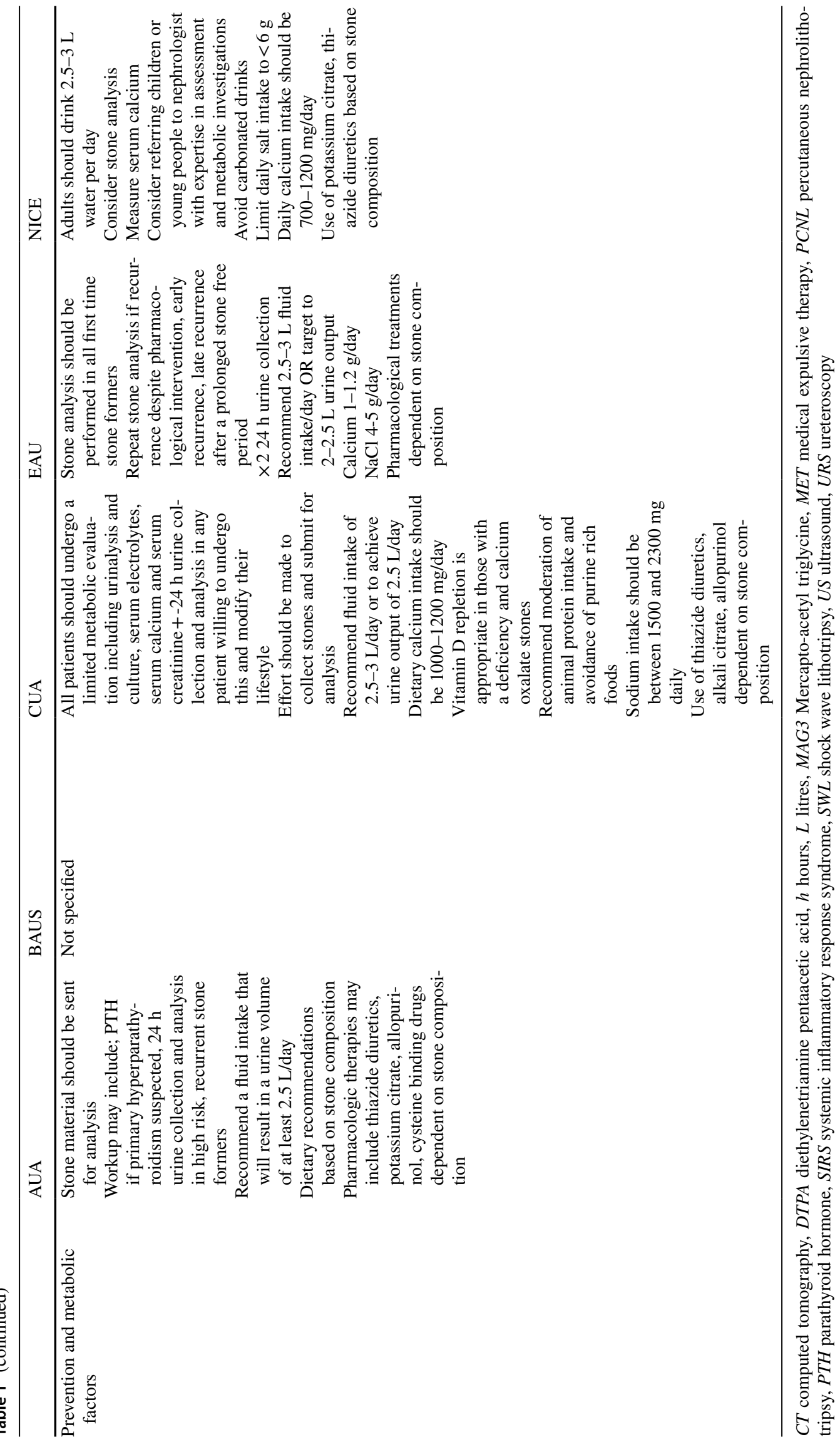




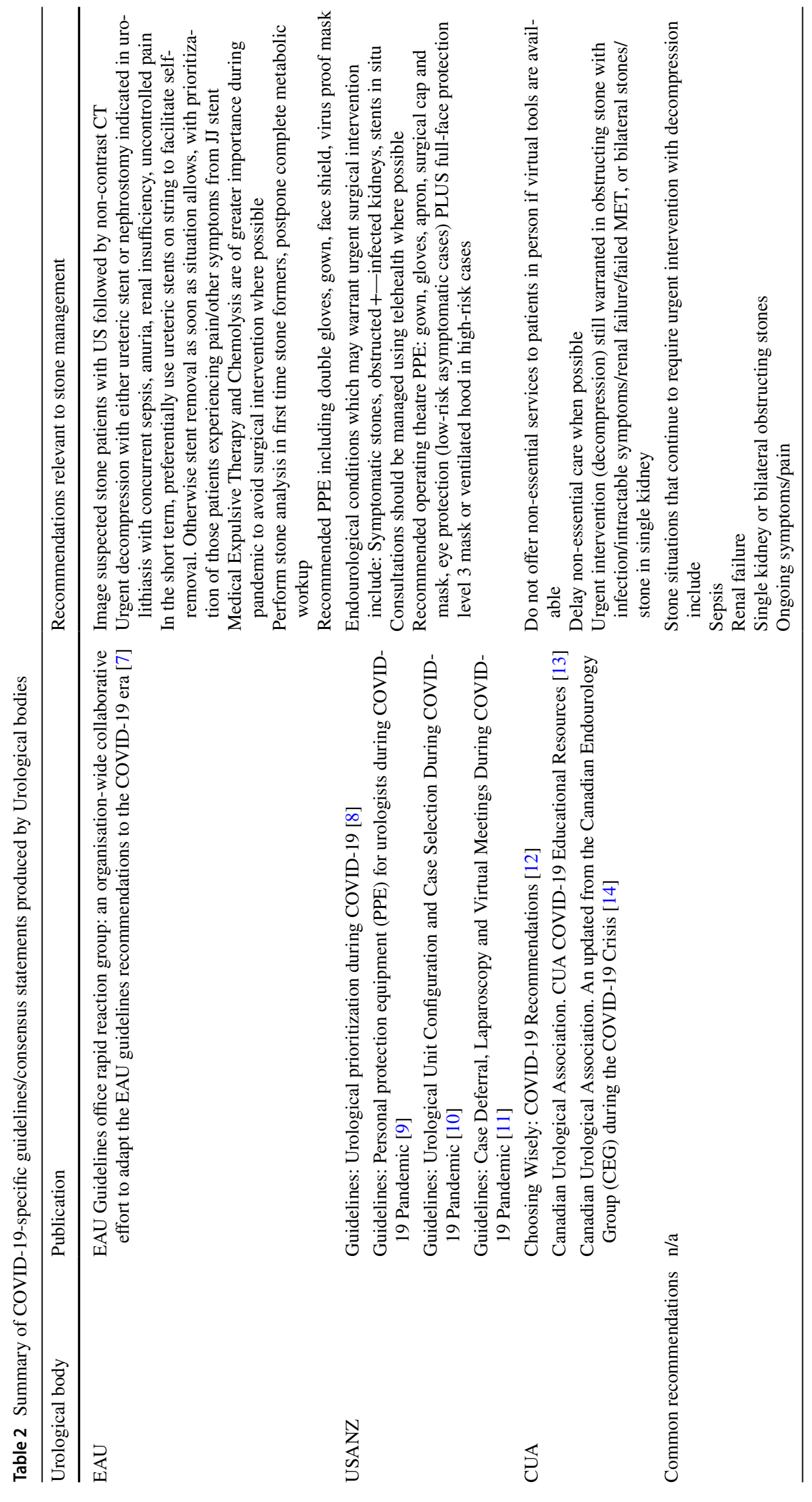


Table 3 Summary of literature relevant to urolithiasis management during COVID-19

\begin{tabular}{lll}
\hline Paper & Publication date & Level of evidence \\
\hline Ficarra et al. [15] & $23 / 3 / 2020$ & Expert OPINION \\
Simonato et al. [16] & $30 / 3 / 2020$ & Expert opinion/na \\
Goldman et al. [17] & $3 / 4 / 2020$ & Expert opinion \\
Katz et al. [18] & $3 / 4 / 2020$ & Expert opinion \\
Puliatti et al. [19] & $6 / 4 / 2020$ & Narrative review \\
Carneiro et al. [20] & $9 / 4 / 2020$ & Narrative review
\end{tabular}

Ho et al. [21] 14/4/2020 Narrative review

Recommendations or findings on urolithiasis in COVID-19

Treat infected/obstructed with stent or nephrostomy Consider ureteral stenting under local anaesthesia if possible

Imaging only to be performed in renal colic refractory to medical treatment

Management with percutaneous nephrostomy or ureteric stenting, perform under local anaesthesia where possible

Procedures for infected and obstructed stones (Tier 0 - threat to life if not performed) and ureteral stones (Tier 1 - threat of permanent dysfunction to extremity or organ) to continue as scheduled

Consider performing flexible cystoscopy and ureteric stent removal without delay to minimise risks of encrustation/UTIs/ retained or forgotten stent

Consider placing ureteral stents or nephrostomy under local anaesthesia if possible

All procedures for urolithiasis should be suspended except for emergencies (infected obstructed, obstructed solitary kidney, bilateral obstruction, acute renal failure, refractory pain)

For infected obstructed stone preferable opt for ureteric stent insertion under spinal anaesthesia, with bedside US guided percutaneous nephrostomy an alternative

Perform primary ureterolithotripsy where safe and possible, utilising a stent with externalised strings to facilitate outpatient removal

Patients with pre-existing ureteric stent in situ should remain with stent in situ for as long as possible

Ureteric stent or nephrostomy insertion for infected obstructed stones remains an emergency, non-deferrable procedure

Perform stenting/nephrostomy under local anaesthesia wherever possible

Consider increased use of stents on strings to avoid additional hospital/procedure attendances wherever possible

References an additional article (Ling et al.) which documents identification of COVID-19 in urine

Consider intervention for obstruction/infection - ureteral stent or nephrostomy, consider performing under LA, however if not possible this is considered an emergent procedure requiring intervention

Most existing stents may undergo simple stent removal with even up to 6-12 months in situ, endoscopic management of stents is possible in most patients up to 30 months of indwelling time

Patients with renal colic should be managed as conservatively as possible

In the case of an obstructed infected kidney, only decompression of the system is recommended, either by stenting or nephrostomy

Ureteric stenting is preferable to nephrostomy due to risk of inadvertent removal of nephrostomy and likely long delay to subsequent lithotripsy

Where possible place ureteric stent or nephrostomy under local anaesthesia to spare a ventilator

Pre-existing indwelling ureteric stents may be left 6-12 months, however, stent indwelling time should be considered in the prioritization process.'

Consider 'pulse antibiotics' in patients with an indwelling stent to reduce risk of urosepsis and requirement of a ventilator

Treat only high priority and emergency cases surgically

A mobile c-arm fluoroscopic x-ray system should be available in any dedicated COVID OR 
Table 3 (continued)

\begin{tabular}{|c|c|c|c|}
\hline Paper & Publication date & Level of evidence & Recommendations or findings on urolithiasis in COVID-19 \\
\hline Pang et al. [25] & $24 / 4 / 2020$ & Case report & $\begin{array}{l}\text { Includes a case report of asymptomatic COVID-19 in a patient } \\
\text { presenting with ureteric calculus }\end{array}$ \\
\hline Ribal et al. [7] & $8 / 5 / 2020$ & Expert opinion and recommendations & $\begin{array}{l}\text { Sepsis and renal failure remain indications for urgent interven- } \\
\text { tion to decompress with ureteric stenting or nephrostomy } \\
\text { Greater utilization of medical expulsive therapy and chemolysis } \\
\text { may be appropriate } \\
\text { Remove indwelling ureteric stents as soon as the situation allows }\end{array}$ \\
\hline Novara et al. [26] & $14 / 05 / 2020$ & Observational cross sectional study & $\begin{array}{l}\text { Identified decrease in total emergency urological presentations, } \\
\text { but increase in immediate JJ stent placement or lithotripsy, } \\
\text { attributed to the need to resolve pain or sepsis and reduce } \\
\text { likelihood of further presentations }\end{array}$ \\
\hline Hughes et al. [27] & 20/05/2020 & Narrative review & $\begin{array}{l}\text { Prioritise intervention in stone cases where there is concurrent } \\
\text { sepsis. Renal failure or stent in situ (justification for intervening } \\
\text { for those with stents in situ being that encrustation observed in } \\
76.3 \% \text { of cases left in situ }>12 \text { weeks) } \\
\text { Use alternatives to ureteroscopy/PCNL such as ESWL wherever } \\
\text { possible } \\
\text { Use stents on strings where possible; } 10 \% \text { risk of premature } \\
\text { dislodgement, however, this is not shown to be associated with } \\
\text { adverse outcome }\end{array}$ \\
\hline Porreca et al. [28] & $20 / 5 / 2020$ & Observational study & $\begin{array}{l}\text { Observed a reduction in emergency urological presentations } \\
\text { including urolithiasis patients (renal colic presentations) during } \\
\text { COVID-19 compared to a pre-COVID reference week }\end{array}$ \\
\hline Chan VW et al. [29] & $27 / 05 / 2020$ & $\begin{array}{l}\text { Systematic Review of Urological } \\
\text { Manifestations of COVID-19 }\end{array}$ & $\begin{array}{l}\text { Identified one study where COVID-19 was detected in Urine } \\
\text { (Peng et al.) }\end{array}$ \\
\hline
\end{tabular}

of COVID-19 rather than specifically reviewing stone disease during COVID-19. As in the guidelines, the literature findings were consistent that urolithiasis with concurrent urosepsis or renal failure is a urological emergency and so continues to warrant urgent intervention within the pandemic environment. Recommendations were also consistent that either ureteric stenting or nephrostomy is an appropriate management strategy for the infected obstructed stone during COVID-19; however, where possible these should be performed under local anaesthesia to reduce use on general anaesthesia and ventilators. No other recommendation was consistent across all papers.

\section{Discussion}

\section{Safety of healthcare workers}

Protecting healthcare workers from infection is of first priority during a pandemic, not only to minimise the risk of the disease and its sequelae to that individual, but also to prevent further transmission within the healthcare environment and external community, and to enable the maximum number of people to continue providing care in a strained healthcare system.

Given there is currently no known vaccination and limited effective treatments outside of supportive care, the only existing strategy for prevention of infection for workers in the healthcare environment is through the use of adequate PPE. To date, COVID-19 has been identified to be present and potentially transmissible in bodily fluids including respiratory secretions, faeces, blood and urine as well as direct contact with surfaces, with some studies finding the virus to survive on inanimate surfaces for up to $72 \mathrm{~h} * *$ [30-33]. Thus, at a minimum, PPE for working directly with potential COVID-19-positive patients should include gown, gloves, eye protection and mask, ideally a N95 mask. Where there is a shortage of N95 or equivalent masks, these should be reserved for use during potentially aerosol-generating procedures. Some guidelines suggest powered air-purifying respirators, although it seems unlikely there are sufficient supplies of these to recommend, they are utilised by all the clinicians [33].

For urologist, the risk of contracting COVID-19 from exposure to urine is thought to be lower than that from other bodily fluids, but isolation of intact infectious virus (not simply virus RNA particles) from urine has been confirmed [34], so nevertheless remains a potential risk to providers performing endoscopic urological interventions such as stenting. Therefore, in addition to mask and eye protection, we would recommend waterproof gumboots and shoe covers to be worn. 


\section{Conservations of resources}

\section{Minimising hospital attendance and length of stay}

Due to the highly infectious nature and exponential spread of the COVID-19 virus, combined with estimated rates of severe and critical illness of $13.8 \%$ and $6.1 \%$, respectively, there is significant risk that need for inpatient care will overwhelm available resources, this having already occurred in locations such as Iran and Italy. Despite this pressure, emergent non-COVID-19 conditions will continue to occur during the pandemic and need to be triaged and managed. For those presenting with acute manifestations of urolithiasis such as renal colic and infected or obstructed stones, choice of treatment has the potential to significantly affect length of stay in hospital.

While renal colic can frequently be managed conservatively with analgesia enabling patients to be discharged home, these patients are prone to representation, especially those with larger and more proximal stones at initial presentation. There is an associated risk of COVID-19 infection at each healthcare encounter in addition to the potential to develop concurrent urinary tract infection or urosepsis in the interim. A crucial part of deciding which patients are appropriate for outpatient conservative management is stone size and location, with non-contrast CT the established gold standard in imaging to assess this. However, waiting for imaging may contribute to an increased length of stay in the emergency department, a high-risk environment for transmission with significant bed availability pressures.

Therefore, we would recommend that during the extreme pandemic environment, patients presenting with symptoms clinically suspicious for renal colic but without evidence of infection or renal failure may be discharged if pain is adequately controlled, with urgent inpatient imaging performed only in the setting of sepsis, renal failure, uncontrolled pain or a repeat presentation. In patients not meeting these criteria, diagnosis may be confirmed or excluded utilising outpatient imaging services with appropriate contact and distancing precautions where this is able to be safely accessed.

In patients requiring intervention, existing information is unanimous that an infected or obstructed system remains a urological emergency requiring immediate decompression, with ureteric stenting and percutaneous nephrostomy considered equivalent. During the pandemic, this should be done under local anaesthesia wherever possible. Some have speculated that ureteric stenting may be preferable to nephrostomy given the potential for accidental removal of nephrostomy during any delay to definite treatment (which is predicted to be more frequent in the pandemic setting) [23]. In reality, this will need to be evaluated on an individual basis, considering both clinical factors such as anticoagulation status and availability of each procedure, ability to tolerate either procedure under local anaesthesia, and social factors including home environment and cognitive ability, for example patients with dementias may be at greater risk of inadvertently dislodging a nephrostomy and, therefore, better suited to stenting.

Finally, for patients with pre-existing stents in situ, the majority of the literature favours accepting an extended stent indwelling time of up to 6-12 months to defer definitive management [22]. We agree but would propose there may be a need for some exceptions to be made in patients known to rapidly and severely encrust stents who would be at risk of loss of renal function from this. For patients who have already undergone stone clearance procedures, self-removal of stent via strings at home is an attractive option that will reduce interaction with the healthcare system during the pandemic. It is important to have a stent register system in place to ensure the patient has removed the stent correctly, this can be achieved via telehealth or phone call. Telehealth consultations can also be utilised in other aspects of outpatient stone management, for example review of serial imaging in stone clinics, to reduce patients' number of hospital attendances with potential exposure to COVID-19 in the healthcare environment. Additional considerations while these strategies are in place during an ongoing pandemic may include provision of dissolution therapy to all patients with indwelling stents to reduce risk of encrustation, and consideration of intermittent antibiotic treatment in those prone to infections and urosepsis while stents are in situ as proposed by Proietti et al., referencing prior work by Tenke et al. finding no significant difference between continuous and intermittent levofloxacin treatment in patients with indwelling ureteral stents or nephrostomy $[23,35]$.

Our recommended initial management of the patient presenting with suspected acute renal colic is summarised by the flowchart (Fig. 1), with potential deviations from usual management outside of the pandemic environment highlighted by greyed boxes or asterisks.

\section{Minimising ICU requirement}

Demand on ICU bed and ventilator requirements will be significantly increased by the COVID-10 pandemic, with current evidence reporting up to $12 \%$ of all patients with COVID-19 may require ICU treatment, in particular with regard to ventilatory support. In those requiring ventilatory support, this may be for a prolonged time course in each individual [32]. In some situations, demand may result in the need to requisition ventilators from other areas such as theatres.

Patients presenting with urolithiasis may compete for these resources in the setting of urosepsis and severe obstructive renal failure where interventions are performed under general anaesthesia. Decompression with ureteric 


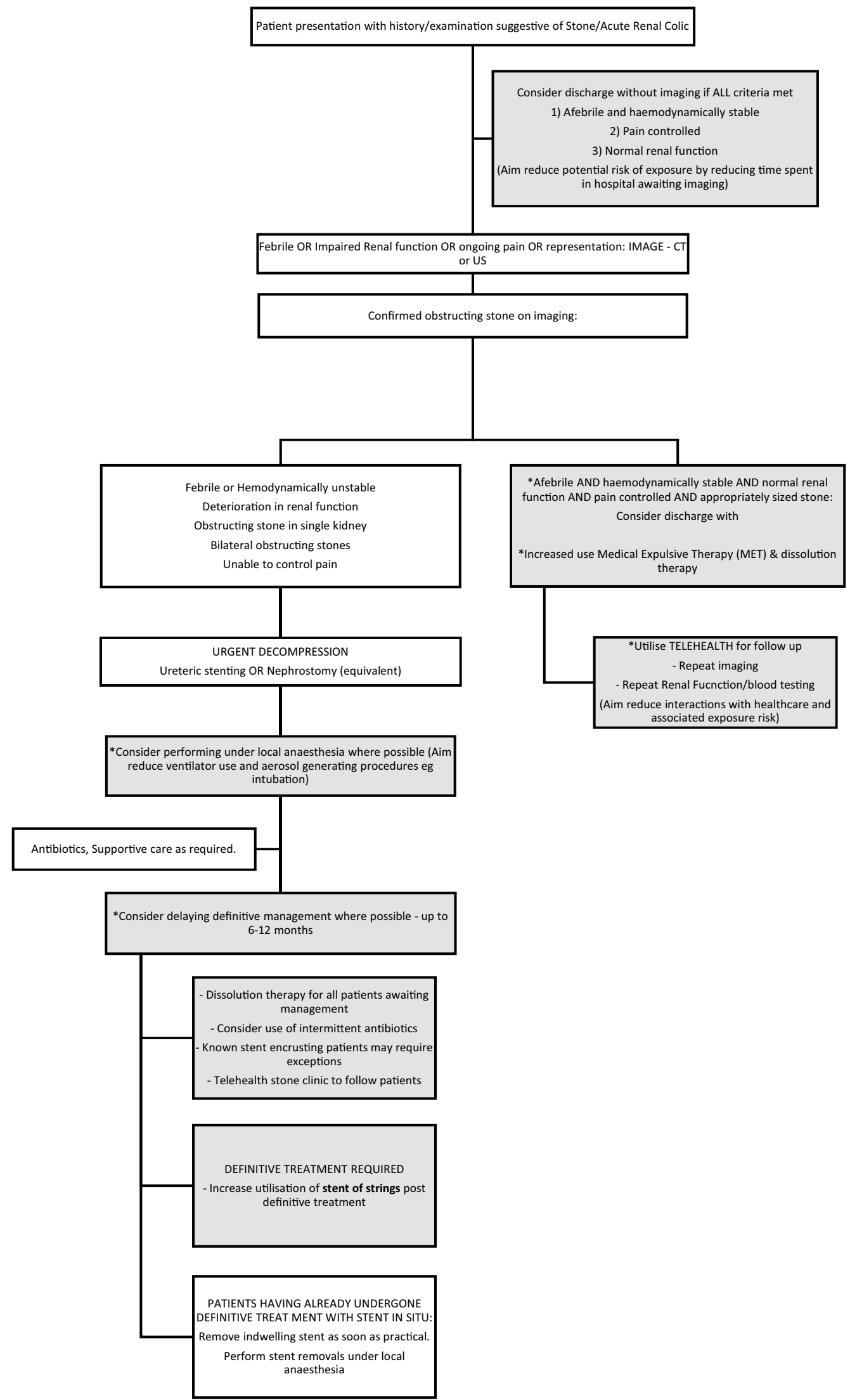

Fig. 1 Recommended initial management of the acute stone presentation during COVID-19 pandemic conditions 
stenting is likely to account for the majority of urolithiasis work during the pandemic and as above should be performed under local anaesthesia wherever possible to reduce demand on ventilators.

In those patients presenting with sepsis, little can be done to reduce their risk of requiring ICU support outside of diligence in collecting urine and blood cultures, and ensuring antibiotic treatment is timely and tailored accordingly. As above, in those patients with ureteric stents or nephrostomy left in situ for a prolonged period of time, intermittent antibiotic treatment may be useful to reduce risk of progression to urosepsis [23].

\section{Conservation of PPE}

Despite the critical role of PPE in protecting healthcare staff, it has already been demonstrated that current global supply is not meeting demand and many locations have already been forced to resort to increasing the accepted length of use of equipment from single use per patient to using the same piece of equipment for entire shifts or even longer [36]. We would recommend a hierarchy of prioritisation of use of PPE for those staff performing aerosolgenerating procedures on known or suspected cases, followed by those otherwise coming into close contact with known or suspected patients, followed by those conducting routine care of all patients.

\section{Anaesthetic implications}

\section{Avoidance of aerosol-generating procedures}

Tracheal intubation, non-invasive ventilation, tracheostomy and bag mask ventilation are all aerosol-generating procedures. Some evidence also suggests aerosols can be generated by laparoscopy, in particular at the time of desufflation [37].

We have already established that in urolithiasis patients, it is likely the vast majority of operative interventions being conducted during the COVID-19 pandemic will be ureteric stent insertions in the setting of sepsis or obstructive renal failure. Where possible, this should be attempted under local anaesthesia, with conscious sedation an alternative that may help avoid need for ventilation.

In those still requiring general anaesthesia, anaesthetists should have prioritised use of appropriate PPE including gown, gloves, full-face shield, N95 or equivalent mask. Other recommendations include the use of, exhalation filters, and video laryngoscopy wherever possible, and attempt to best utilising preoxygenation to avoid use of bag/mask ventilation $[38,39]$.

\section{Future considerations}

Alterations to best practice are likely to be accepted in the short term as required under the extenuating circumstances of a pandemic.

When this resolves and usual practice resumes a downstream effect on case load can be anticipated. In urolithiasis patients, this may result in an increased overall number of patients, greater stone burden, and increase in encrusted ureteric stents. This may affect proportion of patients undergoing SWL compared to ureteroscopy/pyeloscopy and lithotripsy to PCNL. In addition, urologists will need to triage timing of management of postponed urolithiasis cases with deferred oncological cases, aided by updated radiologic imaging of stone burden.

It is also important to consider how to approach this population of patients awaiting deferred definitive stone management in the situation where COVID-19 is suppressed in the local area, but pandemic conditions are ongoing elsewhere-should cases be expedited, or continue to be deferred to preserve PPE for a potential second or subsequent wave? In this event, we believe expediting follow-up and definite stone management (under appropriate precautions such as pre-operative COVID-19 testing and isolation of patients) would be of greater benefit, not only by treating existing deferred patients and reducing their risk of complications such as urosepsis and encrustation associated with prolonged deferral, but also in reducing the deferred patient population anticipating further deferred cases with any additional wave of COVID-19. With regard to the need to conserve PPE in anticipation of second or subsequent waves, we would anticipate this to be of lesser concern as where COVID-19 is suppressed sufficiently as to allow management of deferred stone cases, it should also be possible for PPE manufacturing to be sufficiently increased as to both supply immediate demand and produce surplus to be stockpiled for the event of a future wave.

\section{Conclusion}

In conclusion, it is unavoidable that many patients with urolithiasis will continue to require management during the COVID-19 pandemic. However, it is likely possibly to limit interventions to those patients requiring urgent decompression of the collecting system in the setting of obstruction and or infection, where both general guidelines and COVID-19 specific recommendations from peak Urological bodies are in consensus that decompression of the infected obstructed system remains a Urological emergency requiring immediate intervention. Wherever possible this should be attempted under local anaesthesia utilising either ureteric stenting or percutaneous nephrostomy. Currently available guidelines 
and existing literature suggest either procedure is equally appropriate, and choice should be based on individual consideration of patient and institution factors. Regarding definitive management of urolithiasis, for the duration of the pandemic patients and Urologists will likely need to accept short-term changes to best practice, in particular deferral of definitive stone clearance procedures and accepting an increased length of indwelling stent time.

Author contributions Heijkoop conducted the review and wrote the manuscript. Galiabovitch acted as a second reviewer of the included papers. York and Webb acted as supervisors of the project. All the authors reviewed and approved the final manuscript prior to submission.

Funding No funding was received for this project.

Availability of data and material Data are available from the corresponding author on request.

\section{Compliance with ethical standards}

Conflict of interest No conflicts of interest to declare.

Consent to participate Not applicable-review of literature.

Consent for publication All the authors reviewed and approved the final manuscript prior to submission.

Ethics approval Not applicable—review of literature.

\section{References}

1. Pearle MS, Goldfarb DS, Assimas DG, Curhan G, Denu-Ciocca CJ, Matlaga BR et al. (2014) Medical management of Kidney Stones: AUA guidelines. Am Urol Assoc Guid. Accessed from https://www.auanet.org/guidelines 10/4/2020

2. Assimos D, Krambock A, Miller NL, Monga M, Murad MH, Nelson CP (2016) Surgical management of stones: American Urological Association/Endourological society guidelines. Am Urolog Assoc. Accessed from https://www.auanet.org/guidelines $10 / 4 / 2020$

3. Tsiotras A, Daron Smith R, Pearce I, O’Flynn, Wiseman O (2018) British Association of Urological Surgeons standard for management of acute ureteric colic. J Clin Urol 11(1):58s-61. Accessed from https://www.baus.org.uk/professionals/baus_business/publi cations/133/guidelines_for_the_management_of_acute_ureteric_ colic/ 10/4/2020

4. Dion M, Ankawi G, Chew B, Paterson R, Sultan N Hoddinott $P$ et al. (2016) CUA guideline on the evaluation and medical management of the kidney stone patient-2016 update. Can Urol Assoc. Accessed from https://www.cua.org/en/guidelines $11 / 4 / 2020$

5. Turk C, Neisius A, Seitz DC, Skolarikos A, Tepeler A, Thomas K (2017) EAU guidelines on urolithiasis. EAU. Accessed from https ://uroweb.org/guideline/urolithiasis/ 10/4/2020

6. Renal and ureteric stones: assessment and management. NICE Guid. Accessed from https://www.nice.org.uk/guidance/publishe d?type=csg,cg,mpg,ph,sg,sc 10/4/2020
7. EAU Guidelines office rapid reaction group: an organisationwide collaborative effort to adapt the EAU guidelines recommendations to the COVID-19 era. EAU. Available from https ://uroweb.org/individual-guidelines/rapid-reaction-recommenda tions/. Accessed 22 Aug 2020

8. Guidelines: urological prioritization during COVID-19. USANZ. 25/3/2020

9. Guidelines: personal protection equipment (PPE) for urologists during COVID-19 Pandemic. USANZ. 30/3/2020.

10. Guidelines: urological unit configuration and case selection during COVID-19 Pandemic. USANZ. 05/04/2020

11. Guidelines: case deferral, laparoscopy and virtual meetings during COVID-19 Pandemic. USANZ. 19/04/2020

12. Choosing wisely: COVID-19 Recommendations. Can Urol Assoc. $1 / 4 / 2020$

13. Canadian Urological Association (2020) CUA COVID-19 educational resources [Internet]. Canada: Canadian Urological Association; cited 8/6/2020 Available from: https://mailchi.mp/cua/covid 19resources

14. Canadian Urological Association (2020) An updated from the canadian endourology group (CEG) during the COVID-19 Crisis [Internet]. Canada: Canadian Urological Association; cited 8/6/2020. Available from: https://www.cuasection3credits.org/ CEG

15. Ficarra V, Novara G, Abrate A, Bartoletti R, Crestani A, DeNunzio C (2020) Urology practice during COVID-19 pandemic. Urologica e Nefrologica 72(3):369-375. https://doi.org/10.23736 150393-2249.20.03846-1

16. Simonato A, Giannarini G, Abrate A, Bartoletti R, Crestani A, DeNunzio C (2020) Pathways for urology patients during the COVID-19 pandemic. Urologica e Nefrologica. https://doi. org/10.1111/BJU.15061

17. Goldman HB, Haber GP (2019) Recommendations for Tiered Stratification of Urologic Surgery Urgency in the COVID-19 Era. J Urol. https://doi.org/10.1097/JU.0000000000001067

18. Katz EG, Bukavina L, Stensland KS, Ponsky L, Mandeville JA, MacLachlan LS, Moinzadeh A, Sorcini A, Chang SS, Zaid HB (2019) Triaging office-based urologic procedures during the COVID-19 pandemic. J Urol. https://doi.org/10.1097/JU.00000 00000001034

19. Puliatti S, Eissa A, Eissa R, Amato M, Mazzone E, Dell'Oglio P et al (2020) COVID-19 and Urology: A comprehensive review of the literature. BJUI accepted. https://doi.org/10.1111/BJU.15071

20. Carneiro A, Wroclawski ML, Nahar B, Soares A, Cardoso AP, Kim NJ, Carvalho FT (2020) Impact of the COVID-19 Pandemic on the Urologists clinical practice in Brazil: a management guideline proposal for low an middle Income countries during the crisis period. Int Br J Urol 9:46

21. Ho HC, Hughes T, Bozlu M, Kadioglu A, Somani PK (2020) What do Urologists need to know: Diagnosis, treatment and follow-up during COVID-19 pandemic. Turk J. Urol. 46(3):169-177

22. Stensland KD, Morgan TM, Moinzadeh A, Lee CT, Briganti A, Catto J et al (2020) Considerations in the triage of Urologic Surgeries during the COVID-19 Pandemic. Eur Urol 77(6):663-666. https://doi.org/10.1016/j.eururo.2020.03.027

23. Proietti S, Gaboardi F, Giuto G (2020) Endourological stone management in the era of the COVID-19. Eur Urol. 78(2):131-133. https://doi.org/10.1016/j.eururo.2020.03.042

24. Metzler IS, Sorensen MD, Sweet RM, Harper JD (2020) Stone care triage during COVID-19 at the University of Washington. J. Endourol. 34(5):539-540

25. Pang KH, Osman MI. Asymptomatic COVID-19 Infection in a patient evaluated for Ureteric Colic: radiological findings and impact on management. Urology. https://doi.org/10.1016/j.urolo gy.2020.04.56 
26. Novara G, Bartoletti R, Crestoni A, DeNunzio C, Durant J, Gregori A et al. Impact of the COVID-19 pandemic on urological practice in emergency departments in Italy. BJU Int. https://doi. org/10.1111/bju.15107

27. Hughes T, Ho HC, Shariat SF, Somani BH (2019) Where do Urologists stand in the era of novel coronavirus 2019 disease. Curr Opinion Urol. 30(4):610-616

28. Porreca A, Colicchia M, D'Agostino D, Amenta M, Corsaro A, Zaramello $S$ et al (2020) Urology in the time of Coronavirus: Reduced Access to Urgent and Emergent Urological Care during the Coronavirus Disease 2019 outbreak in Italy. Urol Int 104:631-636

29. Chan VW, Chiu PK, Yee CHJ, Yuan Y, Ng CF, Teoh JY (2020) A systematic review on COVID-19: urological manifestations, viral RNA detection and special considerations in urological conditions. World J Urol. https://doi.org/10.1007/s00345-020-03246-4

30. Guo Y-R, Cao Q-D, Hong Z-S, Tan Y-Y, Chen S-D, Jin HD et al (2020) The origin, transmission and clinical therapies on coronavirus disease 2019 (COVID-19) outbreak - an update on the status. Mil Med Res 7:11. https://doi.org/10.1186/s40779-02000240-0

31. Ozma MA, Maroufi P, Khodadadi E, Kose S, Esposito I, Ganbarov K (2020) Clinical manifestation, diagnosis, prevention and control of SARS-COV-2 (COVID-19) during the outbreak period. Infez Med. 28(2):153-165

32. Phua J, Weng L, Ling L, Egi M, Lim CM, Divatia JV (2020) Intensive care management of coronavirus disease 2019 (COVID-19): challenges and recommendations. Lancet Respir Med 8:506-517

33. Ling Y, Xu S, Lin Y, Tian D, Zhu ZQ, Dai FH et al (2020) Persistence and clearance of viral RNA in 20129 novel coronavirus disease rehabilitation patients. Chin Med J. https://doi.org/10.1097/ CM9.0000000000000774
34. Sun J, Zhu A, Li H, Zheng K, Zhen Z, Zhao C et al (2020) Isolation of infectious SARS-CoV-2 from urine of a COVID-19 patient. Emerg Microb Infect 9:1 Available from https://www.tandfonlin e.com/doi/full/10.1080/22221751.2020.1760144. Accessed 22 Aug 2020

35. Tenke P, Kovacs B, Benko R, Ashaber D, Nagy E (2006) Continuous versus intermittent levofloxacin treatment in complicated urinary tract infections caused by urinary obstruction temporarily relieved by foreign body insertion. Int J Antimicrob Agents 28S:S82-S85

36. Tabah A, Ramanan M, Laupland KB, Buetti N, Cortegiani A et al (2020) Personal protective equipment and intensive care healthcare worker safety in the COVID-19 era (PPE-SAFE): an international survey. J Crit Care 59:70-75

37. RACS Clinical Expert COVID-19 Working Group. Guidelines for safe surgery: open versus laparoscopic-a rapid review commissioned by RACS. R Aust Coll Surg. 2020. Available from: https ://umbraco.surgeons.org/media/5214/2020-04-15-recommenda tions-on-safe-surgery-laparoscopic-vs-open.pdf. Accessed 22 Aug 2020

38. Wax RS, Christian MD (2020) Practical recommendations for critical care and anaesthesiology teams caring for novel coronavirus (2019-nCov) patients. Can J Anaesth 12:1-9

39. Yang M, Dong H, Whihang Lu (2020) Role of anaesthesiologists during the coronavirus disease 2019 outbreak in China. Br J Anaesth 124(6):660-669. https://doi.org/10.1016/j. bja.2020.03.022

Publisher's Note Springer Nature remains neutral with regard to jurisdictional claims in published maps and institutional affiliations. 